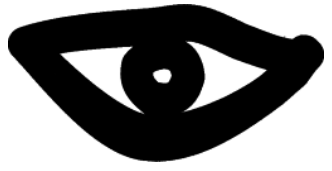

\title{
Una aproximación a los estilos educativos parentales como prácticas socializadoras
}

\section{An approach to parenting styles as socialization practices}

\author{
Ánxela Murillo Casas, Diana Priegue Caamaño y María do Carmen Cambeiro Lourido \\ Grupo de Investigación ESCULCA-Universidade de Santiago de Compostela
}

\begin{abstract}
Resumen
Con la realización de este trabajo pretendemos alcanzar dos objetivos diferentes si bien estrechamente relacionados: de una parte, analizar las principales clasificaciones realizadas sobre los estilos educativos parentales, partiendo de la visión clásica de Diana Baumnrind y centrándonos, sobre todo, en el modelo cuatripartito de Maccobby y Martin, uno de los más aceptados por la comunidad científica; y de otra, estudiar si disponemos de evidencias empíricas que relacionen un determinado estilo educativo con mayores niveles de desarrollo emocional, social y escolar en la infancia y en la adolescencia.
\end{abstract}

Palabras clave: estilo educativo, familia, socialización, desarrollo

\begin{abstract}
The completion of this work we are to achieve two different objectives although closely related: on the one hand, analyze the main classifications made on parenting styles, based on the classical view of Diana Baumnrind and focusing especially on the four-model Maccobby and Martin, one of the most accepted by the scientific community; otherwise, consider whether we have empirical evidence to link a particular parenting style with higher levels of emotional, social and academic development in childhood and adolescence.

Keywords: parenting style, family, socialization, development.
\end{abstract}

\section{Introducción}

La familia es considerada hoy en día como uno de los contextos de desarrollo más importante para las personas que componen su estructura. Por ello, va a jugar un papel muy relevante en el proceso de potenciación y socialización de todos sus miembros, incluso de los adultos, que por el carácter afectivo-relacional-global del contexto familiar, también se ven sometidos a cambios, intelectuales, sociales y afectivos.

Teniendo en cuenta lo anterior, y considerando la complejidad que encierra la intervención en familia en atención temprana, es necesario considerar como una de las variables más importantes en este proceso los estilos educativos parentales, que si bien en una familia con hijos e hijas sin problemas de desarrollo y/o conducta ya son de por sí considerados un pilar básico de un buen funcionamiento y evolución de los niños y niñas, máxime cuando se trata de menores con alguna dificultad añadida.

En este sentido, a lo largo de las últimas décadas se han llevado a cabo múltiples investigaciones y estudios que han aportado evidencias empíricas sobre la influencia del contexto familiar en el proceso de desarrollo infantil y adolescente a nivel integral. De este modo, existe un consenso generalizado a la hora de entender a la familia como el primer y principal agente de socialización de los seres humanos.

En otras palabras, los niños forman su personalidad a través de sus experiencias y el contacto con el ambiente, y uno de los escenarios más importantes, sino el principal, es el seno familiar, de ahí que una distorsión en este contexto cambie también de manera significativa la personalidad y el proceso de desarrollo integral de las personas. Desde la infancia, aprendemos a responder de cierta forma ante las situaciones cotidianas de nuestra vida, y si en nuestro contexto más inmediato, la familia, recurren frecuentemente a la violencia o a estrategias basadas en la autoridad y el conflicto, internalizaremos modelos de conducta inapropiados que repercutirán negativamente en nuestro desarrollo posterior.

Todo ello nos sirve de base para comprender no sólo la estructura y génesis de los diferentes estilos educativos empleados por los padres y madres, sino también su influencia y relación con los demás elementos que configuran el sistema familiar en todos los niveles (relaciones familiares, socialización, funciones, organización, estructura, etc.) así como su papel en el desarrollo de los miembros que lo componen.

Los estilos educativos parentales: aproximación a sus aspectos clave

Los estilos de socialización o estilos educativos parentales organizan patrones persistentes de comportamiento de los padres/madres ante distintas formas de actuación de los hijos/as (García y Gracia, 2010; Pérez Alonso-Geta y Cánovas, 2002; Pérez Alonso-Geta, 2012) de modo que permiten clasificar una parte importante de la relación a largo plazo que se establece entre ellos/as y sus hijos/as. Es decir, los estilos educativos familiares son uno de los ejes fundamentales en la dinámica interactiva que establecen padres/madres con sus hijos/as a nivel relacional, de forma que en torno a ellos se distribuyen los valores, creencias, expectativas, etc. (contenido, el qué se transmite), así como la delimitación de los procedimientos y estrategias (formas, cómo se transmite) presentes en la socialización familiar (Pérez Alonso-Geta, 2002). A partir de estos dos factores -contenido y forma-, se establecen diversas tipologías de estilos educativos o pautas de crianza, los cuales influyen 
sobremanera en la configuración de la personalidad de los hijos/as así como en su desarrollo a todos los niveles: educativo, social, emocional, físico, etc.

En este sentido, una posible definición de este concepto es la que lo identifica con una constelación de actitudes hacia el hijo/a que, consideradas conjuntamente, crean un clima emocional el que se expresan las conductas de los padres (Darling y Steinberg, 1993). Dichas conductas tienen como objetivo principal inculcar en los hijos/as un conjunto de valores, creencias, costumbres culturales, así como contribuir al desarrollo de habilidades sociales, pensamiento crítico, independencia, curiosidad, etc.

Quizás una definición más completa y actual sería aquella en la que se afirma que un estilo educativo surge como consecuencia del sistema de interrelaciones que se estructura a partir de las variables presentes en las prácticas educativas de los progenitores y el peso que cada una de ellas ejerce en las relaciones establecidas con sus hijos/as. Así lo manifiestan Aroca, Cánovas y Sahuquillo (2014) en la siguiente definición:

"Conjunto de pautas y prácticas de crianza, cuyo objetivo es la socialización y educación de los hijos, donde interactúan rasgos de personalidad, experiencias pasadas y características personales, tanto personales como filiales, que se contextualizan dentro de un sistema intra, meso y macrofamiliar inmerso, a su vez, en un marco transcultural e histórico determinados” (Aroca, Cánovas y Sahuquillo, 2014, p. 195).

Teniendo en cuenta el discurso anterior, las investigaciones pioneras sobre la socialización parental, de la mano de Diana Baumrind (1967), distinguían únicamente tres estilos de socialización: autorizativo o democrático, autoritario y permisivo. No obstante, Maccoby y Martin (1983), años más tarde, y basándose en esta primera clasificación, propusieron medir los estilos educativos a partir de las dos dimensiones teóricamente independientes: exigencia y responsividad. Combinando las dos dimensiones y considerando el grado en que los padres y madres actúan en cada una, se desarrolló el modelo cuatripartito de socialización parental, que distingue entre: estilo autorizativo o democrático, estilo negligente, estilo indulgente y estilo autoritario. Es decir, el inicial estilo permisivo del modelo de Baumrind (1967) se desagregó en dos estilos diferentes: el indulgente y el negligente, los cuales se relacionan de forma muy distinta con el ajuste psicosocial de los hijos/as (Pérez Alonso-Geta, 2012; Musitu y Cava, 2001).

Diana Baumrind es una de las autoras pioneras en lo que se refiere al estudio de los estilos parentales de socialización. En este sentido, a lo largo de su trayectoria investigadora ha llevado a cabo múltiples estudios sobre socialización familiar que, siguiendo a Torío et al. (2008, p. 157), son "un punto de referencia fundamental en el campo del apoyo y control parental, la autonomía y el desarrollo de los hijos/as”. Su modelo surge de la combinación de las variables de control, afecto y comunicación, definiendo así tres estilos educativos parentales a través de los cuales los progenitores moldean la conducta de sus hijos/as. Nos referimos a:
-Estilo autoritario (authoritarian discipline): los padres autoritarios valoran la obediencia y la dedicación a las tareas marcadas como una virtud, así como la tradición y el orden. Emplean sobre todo medidas de castigo o fuerza y mantienen a los niños/as en una posición de subordinación restringiendo su autonomía. Se esfuerzan por influir, controlar y evaluar las conductas y actitudes de sus hijos/as a través de patrones rígidos preestablecidos sin la participación de éstos últimos. La comunicación es unidireccional y no facilitan el diálogo. Es el estilo que más repercusiones negativos tiene sobre la socialización de los niños/as, provocando la falta de autonomía personal y de creatividad, una menor competencia social, baja autoestima, inseguridad, poca capacidad de comunicación y demostración de afecto y una pobre interiorización de los valores morales (Torío et al, 2008).

-Estilo permisivo o no restrictivo (permissive discipline): los padres permisivos proporcionan un grado elevado de autonomía a los hijos/as, siempre y cuando no corran peligro. Se comportan de una forma afirmativa, aceptante y benévola hacia los impulsos y acciones de los niños/as, ya que lo que pretenden es liberarlos/as del control y evitar tener que echar mano de la autoridad y el uso de castigos. No son exigentes en lo que se refiere a las expectativas de madurez y responsabilidad, lo que repercute en que los niños/as no suelan asumir compromisos familiares, escolares ni sociales (Muñoz Silva, 2005). Los padres que manifiestan un estilo permisivo presentan altos niveles de comunicación y afecto, sin embargo, no siempre son capaces de marcar límites, de manera que se producen efectos socializadores negativos para la infancia como la prevalencia de conductas agresivas o grados de dependencia elevados.

-Estilo democrático o autorizativo (authoritative discipline): es considerado como el estilo educativo que mayores beneficios aporta a la socialización y desarrollo integral de los hijos/as. Los padres muestran afecto y son sensibles a las necesidades de sus descendientes combinando, al mismo tiempo, cierta firmeza en el mantenimiento de las normas, las cuales son razonadas y negociadas con ellos/as. Es un estilo que se caracteriza por establecer una comunicación bidireccional basada en el diálogo y comprensión mutua, así como también se pone un énfasis especial en equilibrar la responsabilidad social de las acciones de los hijos/as y el desarrollo de su autonomía e independencia (Torío et al, 2008). Algunos de los efectos positivos que produce este estilo son: desarrollo amplio de competencias sociales, índices elevados de autoestima y bienestar psicológico, menor grado de conflictividad en las relaciones paterno-filiales, etc. Es decir, los niños/as cuyos padres/madres emplean estilos democráticos suelen ser hábiles en sus relaciones sociales, interactivos/as, independientes y cariñosos/as.

A partir del punto de referencia de Diana Baumrind, otros autores han realizado sus propias investigaciones en el ámbito de las relaciones parento-filiales y la influencia de las actitudes paternas y maternas hacia sus hijos/as en el desarrollo de éstos. De este modo, en 1983, Maccoby y Martin reformularon el modelo presentado por Baumrind volviendo a interpretar las dimensiones 
básicas propuestas por la citada autora en función de dos aspectos fundamentales: por un lado, el control o exigencia y, por otro, el afecto o sensibilidad. De la combinación de ambos obtuvieron cuatro estilos educativos, ya que descompusieron el estilo permisivo de Baumrind en dos estilos nuevos:

-Estilo autoritario-recíproco, con grados de afecto y control altos. Se correspondería con el estilo democrático identificado por Baumrind. Los padres que utilizan este tipo de estilo son denominados por Maccoby y Martin como "padres con autoridad"

-Estilo autoritario-represivo, con un grado elevado de control y bajo de afecto. Es equivalente al estilo autoritario. Los padres se definían como "padres autocráticos”

-Estilo permisivo-indulgente ("padres indulgentes o permisivos”), con un nivel bajo de control y alto de afecto: Este estilo, siguiendo a Torío et al. (2008), se puede definir en base a tres características de la conducta parental hacia los hijos/as, como la indiferencia ante sus actitudes y conductas (positivas y negativas), la permisividad y la pasividad, compartiendo las características del estilo permisivo propuesto por Baumrind.

-Estilo permisivo-negligente ("padres rechazantes o indiferentes"), con niveles bajos tanto de control como de afecto: Se asocia a un tipo de maltrato. Los padres se caracterizan por la escasa o nula implicación afectiva en los asuntos de los hijos/as y por no asumir la tarea educativa con ellos/as. La permisividad propia de este estilo no es debida a creencias e ideas sobre la educación como ocurre con el estilo permisivo-indulgente, sino a la falta de interés y la negligencia o la comodidad. No invierten apenas tiempo en sus hijos/as y, por ello, prefieren no establecer normas ni control alguno, aunque en ocasiones presentan conductas iracundas y agresivas contra sus hijos/as cuando éstos manifiestan actitudes que rozan lo intolerable. Por otro lado, no tienen inconveniente en complacer las demandas materiales de los hijos/as, comprándoles regalos siempre que sus recursos se lo permitan. En esta clasificación, es el estilo educativo que más consecuencias negativas acarrea para los niños/as, los cuales poseen niveles bajos de autoestima, de capacidades cognitivas y de logros escolares. Además, carecen de autonomía y de competencia para gestionar responsablemente su libertad.

Tabla 1.

Estilos educativos según el modelo de Maccoby y Martin (1983) Fuente: Elaborado a partir de Torío et al. (2008) y Aroca (2010)

\begin{tabular}{|c|c|c|}
\hline & $\begin{array}{l}\text { Nivel alto de } \\
\text { afecto }\end{array}$ & $\begin{array}{l}\text { Nivel bajo de } \\
\text { afecto }\end{array}$ \\
\hline $\begin{array}{c}\text { Control } \\
\text { fuerte }\end{array}$ & $\begin{array}{c}\text { ESTILO } \\
\text { AUTORITARIO-R } \\
\text { ECÍPROCO } \\
\text { (padres con } \\
\text { autoridad) } \\
\end{array}$ & $\begin{array}{c}\text { ESTILO } \\
\text { AUTORITARIO-R } \\
\text { EPRESIVO (padres } \\
\text { autocráticos) }\end{array}$ \\
\hline $\begin{array}{c}\text { Control } \\
\text { laxo o } \\
\text { débil }\end{array}$ & $\begin{array}{c}\text { ESTILO } \\
\text { PERMISIVO- } \\
\text { INDULGENTE } \\
\text { (padres } \\
\text { indulgentes) }\end{array}$ & $\begin{array}{c}\text { ESTILO } \\
\text { PERMISIVO- } \\
\text { NEGLIGENTE } \\
\text { (padres indiferentes) }\end{array}$ \\
\hline
\end{tabular}

\section{¿Cuál es el estilo educativo más adecuado? Algunos estudios al respecto}

Como podemos deducir de nuestro discurso, tradicionalmente se ha asociado el estilo democrático o autorizativo con una influencia más positiva en los hijos e hijas. No obstante, este discurso conviene ser matizado y revisado, ya que las últimas investigaciones han cuestionado las premisas que lo sostienen. En este sentido, en la mayoría blanca anglosajona de los Estados Unidos de América, el estilo autorizativo siempre se ha relacionado con los mejores criterios de ajuste de los hijos/as, si bien estos resultados tradicionalmente no se han podido generalizar a las minorías étnicas de afroamericanos/as, chinos/as o grupos multiétnicos.

Con la partición del estilo permisivo, las excepciones culturales son ahora quizás más considerables, ya que un número destacado de recientes publicaciones sugiere que la relación entre los estilos parentales y el ajuste psicosocial de los hijos/as varía en función del contexto cultural y que, por tanto, el estilo parental de socialización idóneo dependerá del entorno cultural donde éste se desarrolla. Concretamente, recientes estudios llevados a cabo en el contexto español, así como algunos desarrollados en el ámbito anglosajón con minorías étnicas, contradicen la idea de que el estilo autorizativo se asocie siempre a resultados óptimos de la socialización (Martínez, García y Yubero, 2007; Pérez Alonso-Geta, 2012; García y Gracia, 2010; Musitu y García, 2004). Teniendo en cuenta lo anterior, los resultados obtenidos en el estudio llevado a cabo por Pérez Alonso-Geta (2012), en el que se analizó la relación entre los estilos educativos parentales y el ajuste psicosocial de los hijos/as aplicando el modelo cuatripartito de Maccoby y Martin (1983), refuerzan la idea de que en el entorno español, el estilo idóneo para la socialización parental es el permisivo de tipo indulgente, puesto que los hijos/as de estas familias siempre puntuaron en todos los indicadores igual o mejor que los hijos/as de familias autorizativas. Es decir, se concluye que el estilo autorizativo no debería considerarse el prototipo para el contexto español.

En línea con los datos anteriores, en el trabajo de García y Gracia (2010), los resultados obtenidos apoyan la idea de que en España el estilo idóneo para la socialización parental es el indulgente, puesto que los hijos/as de estas familias siempre puntuaron en todos los criterios igual, o incluso mejor, que los hijos de las familias autorizativas, de la misma forma que ocurría en el estudio de Pérez Alonso-Geta (2012). Además de con el autoconcepto, se ha observado una asociación positiva entre estilo parental indulgente y otros criterios como el ajuste psicológico, la competencia personal y menores problemas de conducta.

Estos resultados refuerzan lo que ya resulta bastante claro respecto a la importancia que tienen los componentes de la aceptación/implicación para la socialización familiar:

-El afecto proporciona la cercanía emocional necesaria para que se produzca una relación en la que fluya la comunicación hasta el punto que los hijos/as revelen confiada y espontáneamente aspectos personales e 
íntimos, y exista la cordialidad y confianza necesarias para que la relación con sus padres y madres les proporciones el apoyo necesario para desarrollarse como personas.

-Las relaciones basadas en el afecto, mutua confianza y comunicación abierta facilitan el modelado de la conducta de los hijos/as; el establecimiento de un régimen de reglas claras y bien estructuradas; una comunicación padres-hijos íntima, abierta y espontánea; e incluso una cordialidad mutua que facilita el disfrute del humor (García y Gracia, 2010).

Es decir, siguiendo Montañés y Montañés, Bartolomé y Parra (2008), podemos destacar que los/as adolescentes que disfrutan en su familia de un clima afectivo tienden a mostrar un mejor ajuste y desarrollo psicosocial, más autoestima y mayor competencia conductual y académica. De este modo, se han identificado una serie de factores de riesgo y protección vinculados a la familia en cuanto al desarrollo de conductas agresivas y delictivas en los niños/as y adolescentes así como en problemas de desarrollo psicológico.

Amén de lo anterior, en los resultados del estudio llevado a cabo por Hernández, Gómez, Martín y González (2008) sobre la prevención de la conducta violenta y los estilos educativos de las familias, se identifican una serie de factores, tanto de protección como de riesgo, relacionados con las prácticas educativas de los padres y madres en cuanto a la prevalencia de la violencia en los hijos/as. En concreto, los elementos que pueden incidir en el desarrollo de conductas de tipo violento así como problemas de tipo psicológico en los niños/as y adolescentes, se resumen en los que aparecen reflejados en la siguiente tabla.

Tabla 2.

Prácticas educativas de los progenitores como factores de riesgo y protección en la prevención de la violencia y problemas de ajuste psicosocial

\begin{tabular}{|c|c|}
\hline Factores de riesgo & Factores de protección \\
\hline $\begin{array}{l}\text { Repetir de manera } \\
\text { reiterada las } \\
\text { instrucciones }\end{array}$ & $\begin{array}{l}\text { Reforzar los comportamientos } \\
\text { positivos de los niños y niñas } \\
\text { describiéndoles exhaustivamente } \\
\text { aquello que han hecho bien }\end{array}$ \\
\hline $\begin{array}{l}\text { Aplicar castigo } \\
\text { positivo (pegar) }\end{array}$ & $\begin{array}{l}\text { Aplicar consecuencias } \\
\text { positivas cuando los niños/ } \\
\text { as cumplen sus promesas }\end{array}$ \\
\hline $\begin{array}{c}\text { Aplicar ciertos } \\
\text { castigos negativos }\end{array}$ & $\begin{array}{l}\text { Ser sistemáticos en las normas } \\
\text { y en la aplicación de } \\
\text { consecuencias por } \\
\text { su cumplimiento }\end{array}$ \\
\hline $\begin{array}{l}\text { Aplicar como castigo } \\
\text { el que los niños se } \\
\text { pongan a estudiar }\end{array}$ & $\begin{array}{l}\text { Plantear como objetivo que el } \\
\text { niño/a aprenda a controlar sus } \\
\text { emociones y enseñarle a } \\
\text { responder de manera socialmente } \\
\text { adaptativa cuando dichas } \\
\text { emociones aparezcan }\end{array}$ \\
\hline $\begin{array}{c}\text { Aplicar como } \\
\text { recompensa que los } \\
\text { niños hagan actividades } \\
\text { que durante el resto del } \\
\text { tiempo tienen prohibidas }\end{array}$ & $\begin{array}{c}\text { Mostrar afecto y seguridad } \\
\text { dando lugar a un clima de } \\
\text { confianza y apoyo que haga que } \\
\text { el niño/a se sienta protegido/a y } \\
\text { seguro/a }\end{array}$ \\
\hline
\end{tabular}

Fuente: Elaboración propia a partir de Hernández, Gómez, Martín y González (2008)

\section{Conclusiones}

La familia, como primera unidad de convivencia, debería ser el contexto principal de inicio del proceso educativo, lo que supone desarrollar una capacidad crítica para ejercer la libertad, el respeto y la solidaridad en el entorno de una sociedad diversa e intercultural. Educar es también formar personas con autonomía, responsables y capaces de tomar sus propias decisiones. De este modo, es innegable que la educación es una corresponsabilidad de la familia, la escuela y la sociedad en su conjunto, pero es en el seno familiar donde se "construyen los cimientos que servirán de soporte a los hijos/as a la hora de levantar el edificio de su personalidad” (Cámara y López, 2011, p. 258).

Por ello, los estilos educativos parentales influyen grandemente en la configuración de la personalidad de los hijos/as (autoestima, competencia emocional, etc.); si bien hay que señalar que éstos nunca se dan como tales en estado puro y estricto, ya que en realidad son modelos teóricos que nos acercan a la realidad y en los que aparecen variaciones dentro de amplios márgenes en los que los padres y madres se pueden mover con flexibilidad Es decir, no podemos entender un estilo educativo como una pauta rígida y concreta, sino como una tendencia.

Además, es necesario contextualizar y adaptar cultural y socialmente las prácticas educativas de los progenitores. Lo que en una región puede resultar más beneficioso para el bienestar familiar y el desarrollo de los hijos/as, en otro entorno donde los elementos culturales y/o sociales sean considerablemente diferentes es posible que repercuta de forma negativa o, cuando menos, tenga implicaciones de distinto signo.

Amén de lo anterior, e independientemente de un estilo u otro, a nivel general, las prácticas educativas marcadas por: una rigidez extrema; el autoritarismo; la falta de control y la ausencia de normas; la inconsistencia entre las conductas y actitudes de los miembros de la pareja en cuanto a los hijos/as; y la falta o exceso de afecto y control, son las que actúan como factores de riesgo y determinan en grado sumo la aparición de conductas antisociales y/o delictivas así como problemas de ajuste psicosocial de los hijos/as, con las consiguientes repercusiones para el resto de miembros de la familia.

Es decir, en lugar del establecimiento de conductas y espacios marcados por una autoridad rígida y sin afecto, la base de un correcto desarrollo se encuentra en el ejercicio de estilos educativos caracterizados por un control parental equilibrado y unas relaciones basadas en el afecto, el apoyo, la comunicación, el acompañamiento y la implicación en la vida cotidiana de los hijos/as. Sólo de esta forma se podrá lograr una autoridad legitimada ante los niños/as y adolescentes que se base en el respeto mutuo, la tolerancia, la comprensión y el diálogo. 


\section{Referencias}

Aroca, C. (2010). La violencia filio-parental: una aproximación a sus claves (Tesis doctoral). Valencia: Universidad de Valencia.

Aroca, C.; Cánovas, P. y Sahuquillo, P. (2014). Los estilos educativos. En P. Cánovas y P. M Mateo (coords.), Familias y menores. Retos y propuestas pedagógicas (pp.189-230). Valencia: Tirant Humanidades.

Baumrind, D. (1967). Child care practices anteceding three patterns of preschool behavior. Genetic Psychology Monographs, 75, 43-88.

Cámara, E. y López, J.B. (2011). Estilos de educación en el ámbito familiar. Revista Española de Orientación y Psicopedagogía, 22(3), 257-276.

Darling, N. y Steinberg, L. (1993). Parenting style as context: An integrative model. Psychological Bulletin, 113, 487-496.

García, F. y Gracia, E. (2010). ¿Qué estilo de socialización parental es el idóneo en España? Un estudio con niños y adolescentes de 10 a 14 años. Infancia y aprendizaje, 33(3), 365-384.

Hernández, M., Gómez, I., Martín, M.J., y González, C. (2008). Prevención de la violencia infantil-juvenil: estilos educativos de las familias como factores de protección. International Journal of Psychology and Psychological Therapy, 8, 73-84.

Maccoby, E. y Martin, J. (1983). Socialization in the context of the family: Parent-child interaction. En E. M. Hetherington y P. H. Mussen (Eds.), Handbook of child psychology: Socialization, personality, and social development (pp. 1-101). New York: Wiley.

Martínez, I., García, J. F. y Yubero, S. (2007). Parenting styles and adolescents' self-esteem in Brazil. Psychological Reports, 100, 731-745.
Montañés, M.; Montañés, J.; Bartolomé, R. y Parra, M. (2008). Influencia del contexto familiar en las conductas adolescentes. Ensayos: Revista de la Facultad de Educación de Albacete, 23, 391-408.

Musitu, G.; Estévez, T.; Jiménez, I. y Herrero, J. (2007). Familia y conducta delictiva y violenta en la adolescencia. En S. Yubero et al. (coords.), Convivir con la violencia. Un análisis desde la psicología de la educación de la violencia en nuestra sociedad, pp. 135-150. Cuenca: Ediciones de la Universidad de Castilla-La Mancha.

Musitu, G. y Cava, Ma J. (2001). La familia y la educación. Barcelona: Octaedro.

Musitu, G. y García, J.F. (2004). Consecuencias de la socialización familiar en la cultura española. Psicothema, 16(2), 288-293

Musitu, G., y Cava, M.J. (2001).La familia y la educación. Barcelona: Octaedro.

Panchón, C. (2002). La gestión de la calidad en la acción socioeducativa. Educación Social: Revista de Intervención Socioeducativa, 21, 10-24.

Pérez Alonso-Geta, P. M ${ }^{\mathrm{a}}$ (2002). Crianza y estilos familiares de educación. En E. Gervilla, Educación Familiar. Nuevas relaciones humanas $y$ humanizadoras (pp. 65-80). Madrid: Narcea.

Pérez Alonso-Geta, P. M M (2012). La socialización parental en padres españoles con hijos de 6 a 14 años. Psicothema, 24(3), 371-376.

Pérez-López, J. (2006). Bases de la intervención familiar en atención temprana. En J. Pérez-López y A.G. Brito de la Nuez (coords.), Manual de Atención Temprana (pp. 333-351). Madrid: Pirámide.

Rodrigo, Ma . J. y Palacios, J. (1998) (coords.). Familia y desarrollo humano. Madrid: Alianza Editorial. 